'Escuela de Medicina, Facultad de Medicina y Ciencia, Universidad San Sebastián, Lota 2465, Providencia, Santiago, 7510157, Chile. ${ }^{2}$ Programas para el Futuro, Facultad de Estudios Interdisciplinarios y Escuela de Medicina, Facultad de Ciencias, Universidad Mayor. Santiago, Chile.

${ }^{3}$ Centro de Biología Celular y Biomedicina (CEBICEM), Universidad San Sebastián y Centro de Envejecimiento y Regeneración UC, Pontificia Universidad Católica de Chile. Santiago, Chile. ${ }^{4}$ Departamento de Manejo Integral de Cáncer y otros Tumores, Ministerio de Salud. ${ }^{5}$ Unidad de Coloproctología, Clínica Las Condes. Santiago, Chile. ${ }^{6}$ Departamento de Fisiopatología, Escuela de Medicina, Subdirección de Investigación, Facultad de Cs. Médicas, Universidad de Santiago de Chile. ${ }^{a}$ Nutricionista, Mg. en Salud Pública. ${ }^{b}$ Enfermera. CPhD.

Trabajo no recibió financiamiento. Los autores declaran no tener conflictos de interés.

Recibido el 2 de diciembre de 2019, aceptado el 22 de abril de 2020.

Correspondencia a: Juvenal A. Ríos Leal M.D., M.Sc., Ph.D. Director Diplomado en Medicina Traslacional, Programas para el Futuro, Facultad de Estudios Interdisciplinarios y Escuela de Medicina, Facultad de Ciencias, Universidad Mayor. jriosleal@gmail.com juvenal.rios@mayor.cl

\section{Situación actual del cáncer de colon en Chile: una mirada traslacional}

\author{
JUVENAL A. RÍOS ${ }^{1,2, c}$, M. FRANCISCA BARAKE ${ }^{3, c}$, \\ MARÍA JOSÉ ARCE ${ }^{4, a}$, FRANCISCO LÓPEZ-KÖSTNER ${ }^{5}$, \\ TOMAS P. LABBE ${ }^{6, \mathrm{c}}$, JESSICA VILLENA ${ }^{4, \mathrm{~b}}$, \\ SERGIO BECERRA ${ }^{4}$
}

\section{The present situation of colorectal cancer in Chile}

Colorectal (CRC) is one of the most common types of cancer worldwide. Most tumors develop from an adenoma in a period of 10 to 15 years, but some may appear without previous adenomatous lesions. Seventy-five percent of colorectal cancers are sporadic, 20\% have a family component (first or second-degree relatives with $C R C$ ) and 5\% have a hereditary predisposition with a Mendelian pattern. The epidemiological evolution in the recent years in Chile has a worrisome evolution and the treatment costs of advanced stages are a burden for the healthcare system. We herein highlight the main Chilean medical and scientific contributions on the pathogenesis, early diagnosis, and treatment of $C R C$, which lead to its better understanding, and therefore better management, based on local evidence.

(Rev Med Chile 2020; 148: 858-867)

Key words: Diagnosis; Neoplasm; Therapeutics; Translational Medical Research.

\section{Consideraciones generales}

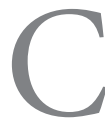

hile aún está lejos de alcanzar la magnitud que representa el cáncer de colon y recto (CCR) de los países desarrollados, sin embargo, el incremento de las tasas de mortalidad en los últimos años genera grandes costos para el sistema. En este sentido, el Ministerio de Salud de Chile (MINSAL) desde el año 2013, asegura el financiamiento del diagnóstico y tratamiento del CCR a través de las Garantías Explícitas en Salud (GES), constituyéndose como el problema de salud (PS) número $70^{1}$. En paralelo a este esfuerzo ministerial, existe una Guía de Práctica Clínica de CCR recientemente actualizada y disponible en el sitio web del MINSAL ${ }^{2}$, la que proporciona un conjunto de recomendaciones destinadas a los equipos de salud como ayuda para seleccionar aquellas opciones terapéuticas más adecuadas basándose en la mejor evidencia científica disponible. Además, existen otros instrumentos regulatorios como protocolos de manejo clínico y orientaciones técnicas en construcción y pronta publicación.

En los inicios de la historia natural del CCR, se describe la aparición de criptas aberrantes que dan lugar a la formación de adenomas microscópicos que, con el paso del tiempo, avanzan hasta la superficie epitelial y dan lugar al pólipo. En la etapa invasora, se realiza la evolución de pólipo a carcinoma, la cual es en general, de forma ordenada y con tendencia al crecimiento circular, invadiendo las diferentes capas de la pared colónica ${ }^{3,4}$. 
La mayoría de los casos de CCR son esporádicos, un quinto tiene un fuerte componente familiar, y una proporción muy menor sigue un patrón genético mendeliano, de éstas las dos variantes más importantes son el:

\section{Sindrome de Lynch (SL)}

Corresponde al 5\% de los CCR y representa el síndrome hereditario con predisposición al CCR más frecuente. Está causado por alteraciones en genes de reparación del ADN (MLH1, MSH2, MSH6). Tiene una edad de aparición temprana (antes de los 50 años) y se localiza preferentemente en el colon derecho ${ }^{3,4}$.

\section{Poliposis adenomatosa familiar (PAF)}

Corresponde al 0,5\% de los CCR. Sigue un patrón de herencia autosómico dominante por mutaciones en el gen APC. Se caracteriza por la presencia de más de 100 pólipos en el colon y recto $^{3,4}$.

El aumento del CCR en países desarrollados es atribuible al envejecimiento poblacional y a la exposición prolongada a factores de riesgo asociados al comportamiento individual, entre ellos, una alimentación no saludable, el tabaquismo, inactividad física y obesidad ${ }^{5}$. Pese a los constantes avances tecnológicos en el abordaje quirúrgico y a la introducción de nuevas terapias antineoplásicas, todo indica que la prevención y la detección precoz seguirán siendo las mejores estrategias para mitigar el incremento del $\mathrm{CCR}^{6}$.

A continuación, presentamos una recopilación de los principales esfuerzos de la academia y hospitales chilenos por mejorar la comprensión y el tratamiento del CCR, mediante una sistematización de la información que aborda los aspectos básicos y clínicos de la investigación local, con una mirada traslacional, entendiendo que este tipo de investigación se realiza con la finalidad de mejorar la salud de las personas.

\section{Investigación}

\section{Epidemiología}

El CCR constituye el tercer tumor maligno más frecuente en la población mundial con una tasa cruda de incidencia de 24,2 por 100.000 habitantes y la segunda causa de muerte por neoplasias con una tasa cruda de mortalidad de 11,5 por 100.000 . De estos, en su mayoría son varones, constituyendo $55 \%$ de los nuevos casos y muertes ${ }^{7}$.En Chile, para el año 2012, se registraron 2.417 casos nuevos de cáncer de colon, recto y ano en ambos sexos. La Tasa Ajustada de Incidencia (TAI) fue levemente superior en hombres que en mujeres (10,8 y 9,6 100.000 habitantes, respectivamente $)^{8}$. No obstante, las proyecciones de incidencia más recientes estimaron para el país 5.914 casos nuevos de CCR en ambos sexos (3.002 en hombres y $2.912 \mathrm{en} \mathrm{mu-}$ jeres), con una TAI de 20,7 por 100.000 habitantes para ambos sexos. El reporte más actualizado de mortalidad del año 2016 calculó una Tasa Anual de Mortalidad (TAM) de 7,19 por 100.000 habitantes para el CC y 1,99 por 100.000 habitantes para el CR. La evolución de las TAM de ambos cánceres aumentó más de 20\% entre los años 2000 a 2016 (Figura 1$)^{9}$.

Asimismo, existen diferencias vinculadas al sexo en ambos cánceres entre los años 2000 a 2016 (Figura 2), y en cuánto a su distribución regional la TAM del cáncer de colon y recto son sustancialmente mayores en la Región del Maule (8,6 por 100.000 habitantes) y en la Región de Aysén (7,05 por 100.000 habitantes) que el promedio nacional (Figura 3$)^{9}$.

\section{Etiopatogenia}

Entre las áreas más importantes en investigación básica, destacan: (i) estudios respecto a la biología tumoral del CCR; (ii) estudios de la frecuencia de mutaciones en genes conocidos para las formas hereditarias de CCR y (iii) estudios de marcadores moleculares predictivos en el contexto de la forma esporádica de CCR.

Sobre la biología tumoral, en el año 2016 el grupo del Dr. Tapia (U. Chile ${ }^{10}$, encontró que la proteína CK2, mediante fosforilación, estabiliza la actividad de la enzima convertidora de endotelina 1c (metaloproteasa de membrana), lo cual aumenta la capacidad invasora y migratoria de un linaje de células de CCR, ubicando a CK2 como posible blanco terapéutico en $\mathrm{CCR}^{10}$. En 2019, el mismo laboratorio comprobó que silmitasertib, una droga previamente probada como inductor de apoptosis para células de cáncer pancreático, también lo hacía en células DLD-1, mediante la inhibición de $\mathrm{CK} 2{ }^{11}$. En otro escenario biológico en el año 2018, se reportó el efecto de las quimioquinas plasmáticas (CCL2, 3 y 4 ) en tejido tumoral de 


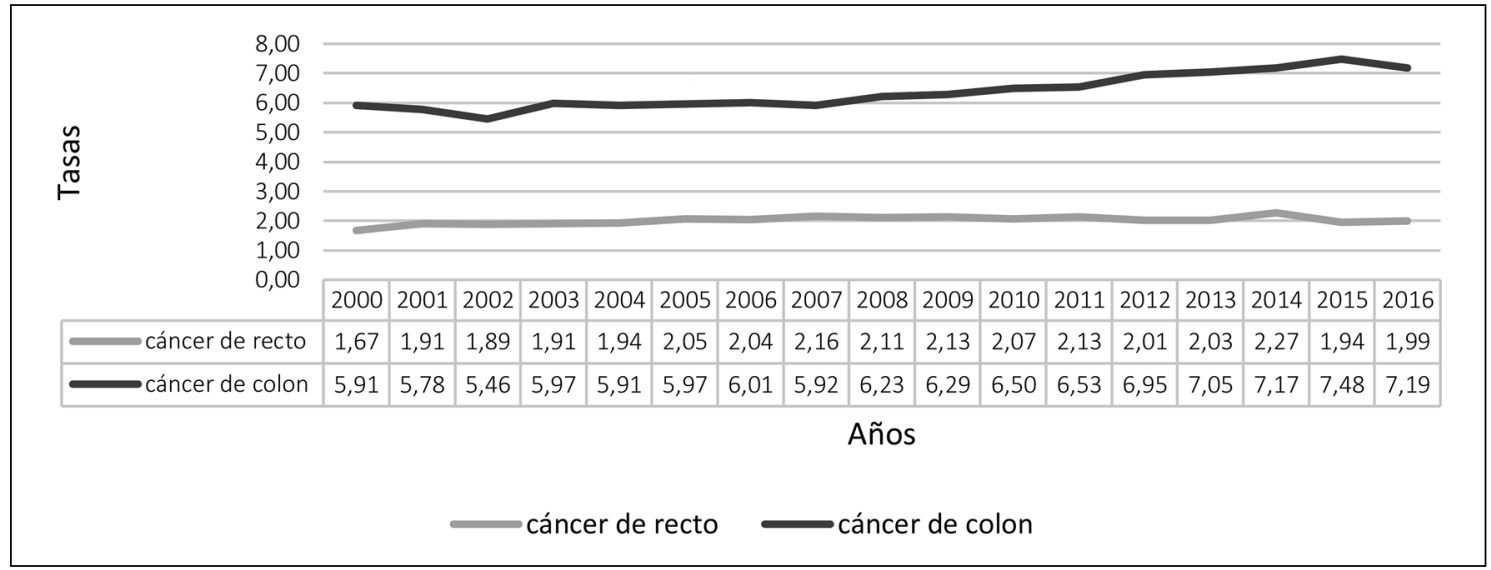

Figura 1. Tasas de mortalidad ajustada del cáncer de colon y recto en Chile durante los años 2000 a 2016 (elaboración propia en base a serie de tumores malignos del DEIS 2000-2016).

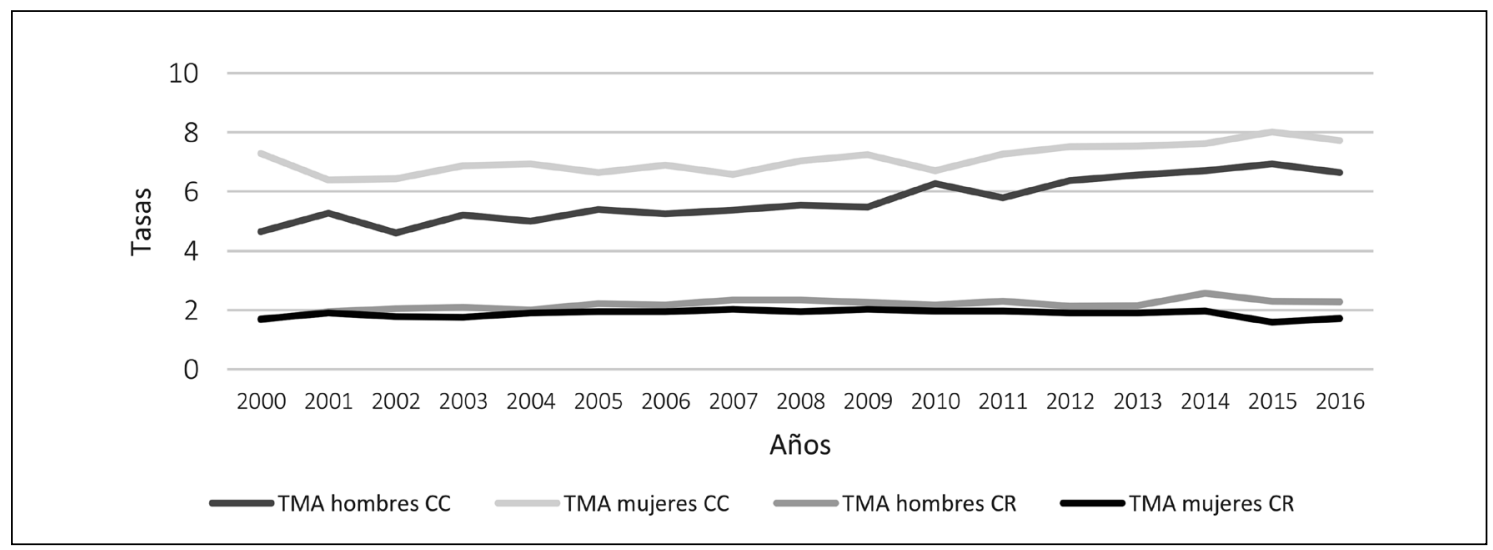

Figura 2. Tasas de mortalidad ajustada del cáncer de colon y recto en Chile por sexo durante los años 2000 a 2016 (elaboración propia en base a serie de tumores malignos del DEIS 2000-2016).

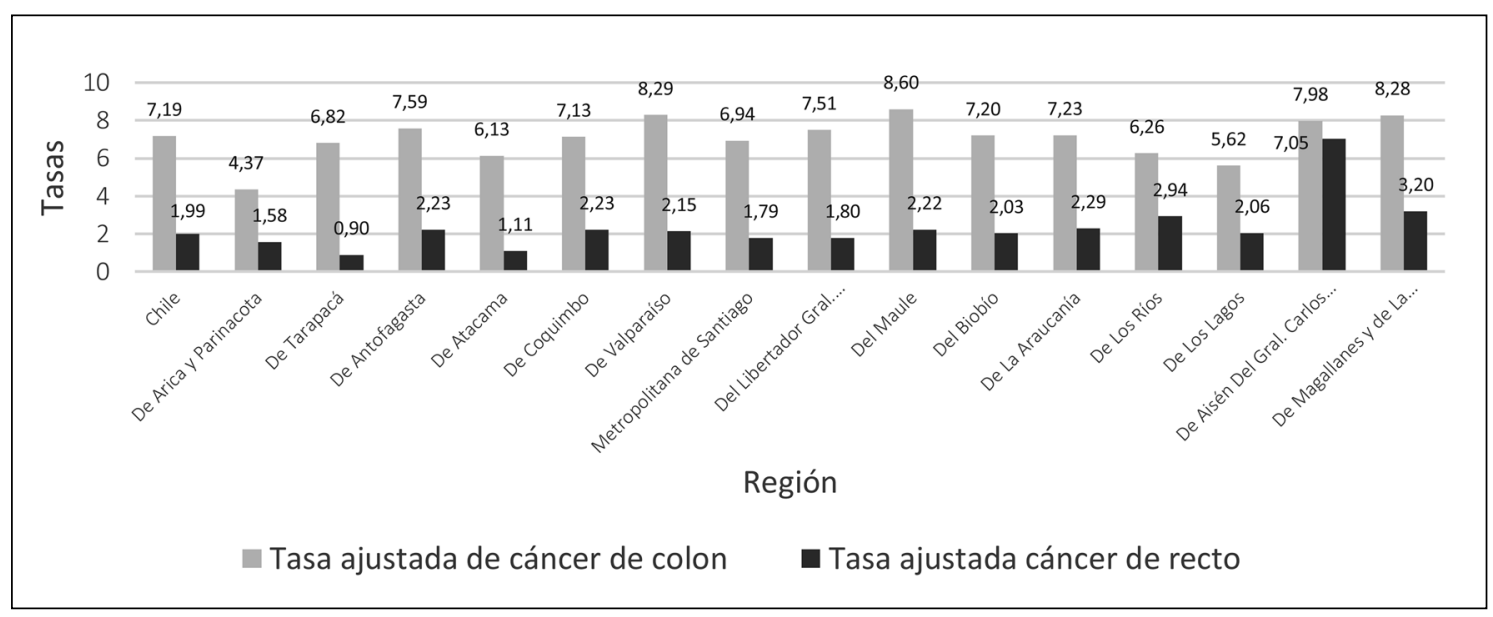

Figura 3. Distribución de la tasa de mortalidad ajustada del cáncer de colon y recto en distintas regiones de Chile durante el año 2016 (Elaboración propia en base a serie de tumores malignos del DEIS 2000-2016). 
cáncer de colon, encontrando que particularmente CCL4 estimula la infiltración de macrófagos, en especial, el tipo tumoral de macrófagos CD163 $+{ }^{12}$.

En cuanto al estudio de los genes de las formas hereditarias, entre los años 2004-2006, el grupo de López-Köstner ${ }^{13}$, hizo un estudio genealógico en pacientes con SL y PAF, identificando en tres familias chilenas, dos mutaciones descritas para el gen MLH1 y APC, de las cuales se sabe que requieren un manejo endoscópico y quirúrgico precoz ${ }^{13}$. Posteriormente, este laboratorio estudió a 21 familias con CCR con criterios de sospecha para SL, identificando mutaciones de los genes MLH1 y MSH2, en el 45\% de ellas. De las 9 mutaciones descritas, solo 2 eran compartidas por población latinoamericana y 2 fueron identificadas como nuevas mutaciones ${ }^{14}$. En la misma línea, el Programa de Genética Humana de la Universidad de Chile, reportó un caso de PAF con una mutación en APC, que tenía 400 pólipos y correspondía a la tercera generación de familiares con $\mathrm{CCR}^{15}$.

El estudio de la etiopatogenia del CCR esporádico ha sido más complejo y ha cambiado mucho en los últimos años, en virtud del explosivo descubrimiento de nuevos mecanismos celulares y moleculares, y por tanto, de nuevos marcadores que contribuyen al diagnóstico de precisión de la enfermedad ${ }^{16}$.

La vía de señalización del receptor de crecimiento epidermal (EGFR) está muy asociada al desarrollo del CCR. El EGFR está involucrado en proliferación, diferenciación, apoptosis y angiogénesis, mediante la vía señalización de RAS/ RAF/MAPK y PI3K/PTEN/AKT. Los inhibidores de EGFR son utilizados en los casos de cáncer metastásico, por lo que es importante saber si hay mutaciones de otras proteínas de la vía del EGFR que le puedan conferir resistencia al tratamiento ${ }^{17}$. En pacientes chilenos con CCR, se ha encontrado una frecuencia de mutaciones que oscila en el gen KRAS entre $28 \%$ a $45 \%^{18-21}$, en BRAF entre $9-14 \%{ }^{19,21}$, PIK3CA entre $16 \%-21 \%^{19,21}$ y $7 \%$ de PTEN además de un 10,7\% de deleciones de este último ${ }^{21}$.

En 2015, el grupo de Solé y col $^{22}$ analizó la infección por citomegalovirus (HCMV) y virus Epstein-Barr (EBV) con parámetros clínicos, histopatológicos, metabólicos tumorales mediante PET-CT y moleculares (KRAS), en pacientes con cáncer rectal localmente avanzado. Al analizar 37 pacientes, se encontró que personas con co- infección (HCMV/EBV) tienen mayor captación metabólica, volumen tumoral y presencia de KRAS mutado, sin embargo, no hubo diferencias en sobrevida global ni en sobrevida libre de progresión.

Respecto de los daños genómicos que ocurren en el desarrollo del CCR, se describen las siguientes rutas: (i) la inestabilidad cromosómica (CIN) donde cambia el número y la estructura del cromosoma, afectando la función de genes represores de tumores, o aumentando el número de copias de protooncogenes; (ii) la inestabilidad microsatelital (MSI) donde la célula es incapaz de corregir deleciones o inserciones en regiones repetitivas del $\mathrm{ADN}$ generando un amplio número de mutaciones y (iii) el fenotipo metilador (CIMP) en el cual los promotores de genes supresores de tumores están hipermetilados y, por lo tanto, inactivos. Se ha mostrado que el $50 \%$ de los pacientes chilenos tienen alta $\mathrm{CIN}^{23}$, entre el $17 \%$ al $27 \%$ tiene alta $\mathrm{MSI}^{19,21,23}$ y por último un $31 \%$ tiene alto grado de $\mathrm{CIMP}^{23}$.

\section{Diagnóstico}

Es un hecho que el CCR puede ser diagnosticado precozmente, incluso en etapas premalignas y que esto incide en la disminución de su incidencia y mortalidad ${ }^{24,25}$. En personas con bajo riesgo de cáncer y asintomáticos se sugiere hacer una prueba de sangre oculta en deposiciones inmunológico (TSODi) anual a partir de los 50 años, prueba que ha mostrado una simulación costo-efectiva favorable y económicamente positiva para nuestro medio nacional ${ }^{26}$. Ante un resultado positivo, se debe complementar con colonoscopía completa, cuya sensibilidad y especificidad es mayor, al igual que los costos, pero es operador dependiente y no está exenta de riesgos.

En el año 2012 se publicó un estudio prospectivo multicéntrico, sometiendo sujetos de bajo riesgo (6.348 sujetos de bajo riesgo en 7 ciudades del país) a un TSODi, prueba que en comparación a la Guayaco no necesita preparación dietaria previa. El TSODi tuvo $77 \%$ de adherencia anual y cerca de $10 \%$ resultó positivo. De estos, $58,6 \%$ se realizó la colonoscopía completa, detectando pólipos en $40,2 \%$. Los adenomas fueron resecados y 1,1\% correspondió a cáncer en etapa localizada ${ }^{27}$. Luego se generó un segundo reporte que consideró una mejoría en el diseño de los protocolos, así como realización de capacitaciones endoscópicas, de anatomía patológica y de biología molecular, bajo 
el alero del Programa de Prevención de Neoplasias Colorrectales (PRENEC) que es una alianza entre Universidad Médica y Dental de Tokyo (UDTM), Clínica Las Condes (CLC) y MINSAL ${ }^{28,29}$. Esta segunda serie siguió a 12.668 pacientes en 3 ciudades sujetos a TSODi, de los cuales hubo 93,9\% de adherencia. De los pacientes con cáncer, 91\% fueron detectados y resueltos en etapas tempranas y además se obtuvieron mejores parámetros endoscópicos y tasas de detección ${ }^{30,31}$.

El mismo grupo de López-Köstner ${ }^{32}$ estudió una serie de casos chilenos con sospecha de SL para validar su diagnóstico, mediante detección de inestabilidad microsatelital (MSI) por inmunohistoquímica (IHQ), demostrándose una buena correlación con el estudio genético, en la medida que se utilice los criterios de Ámsterdam. De este modo, recomiendan el uso de la IHQ para MSI como una técnica que ahorra tiempo y costos y que tiene mayor disponibilidad que los estudios genéticos ${ }^{32}$.

\section{Terapéutica}

En la investigación del tratamiento del CCR existen reportes locales publicados en diversas áreas, las cuales van desde estudios pronósticos, aspectos quirúrgicos y hasta evaluación del rendimiento de nuevas terapias biológicas en nuestra población.

El grupo de la Universidad de Chile - sede Hospital San Borja Arriarán (HSBA) ${ }^{33}$, son quienes han hecho las mayores aportaciones en la evaluación de los resultados inmediatos y alejados de la cirugía del CCR. Una serie con 610 pacientes demostró que, en la resección por patologías colorrectales, con anastomosis colorrectal, la reintervención pre$\mathrm{coz}(\mathrm{RP})$ era más probable en pacientes con bajo estado nutricional medido por albúmina sérica y en los de sexo masculino. Además, la RP aumentó 8 veces el riesgo de infección herida operatoria, tiempo de hospitalización promedio y mortalidad operatoria ${ }^{33}$. La reintervención es, además, factor de riesgo para el reingreso hospitalario, la cual fue de $7 \%$ en un grupo de 791 pacientes. De este modo, la identificación de estos factores de riesgo permite su control y consecuente mitigación, lo que en teoría reduciría los tiempos de hospitalización y los costos asociados, sin embargo, es un problema que aún sigue siendo complejo y claramente multifactorial ${ }^{34}$.

En particular sobre la cirugía de cáncer de co- lon, este mismo grupo mostró tener morbilidad y mortalidad similar a otras series extranjeras, incluso comparándola con centros especializados, pero con una tasa resecabilidad algo menor, probablemente por el mayor tamaño del tumor y ganglios comprometidos, reportados en la cohorte. Además, se encontró que la tasa de sobrevida era significativamente menor en pacientes desde el estadio TNM IIIb de la clasificación TNM 2002. Agregando el TNM en el modelo de regresión, se detectó que la permeación vascular y el número de ganglios positivos eran otros factores pronósticos importantes ${ }^{35}$.

En relación con el cáncer de recto, se habla de "otra enfermedad", el tratamiento quirúrgico es diferente y se asocia con radio quimioterapia preoperatoria y cirugía radical. En 2010 el grupo del HSBA mostró los primeros resultados de este esquema. La neoadyuvancia preoperatoria disminuyó el compromiso transmural y en menor medida el compromiso linfonodal, aumentando la sobrevida al largo plazo $^{36}$. En otro estudio se comparó dos formas de protección de anastomosis colorrectal baja, usando una cecostomía amplia en vez de una ileostomía en asa. Se demostró que ambas técnicas quirúrgicas son comparables en desfuncionalización del tracto colónico distal y morbimortalidad e incluso la ileostomía en asa tuvo mayores tiempos de estadía hospitalaria con mayor número de pacientes con disfunción ${ }^{37}$. En cuanto al abordaje quirúrgico, existe discrepancia entre cirugía abierta y laparoscópica. En 2016 el grupo del Hospital Clínico de la Universidad de Chile publicó su experiencia con un abordaje mixto usando un puerto endoanal en combinación con laparoscopía convencional para resecciones interesfintérica del recto bajo y disección rectal transanal, mostrando ser una opción segura ${ }^{38}$.

Otro avance importante en el tratamiento quirúrgico del CCR ha sido el desarrollo del acceso por video-laparoscopía ya que está asociado a menor estadía hospitalaria y tasa de complicaciones (infección de herida operatoria, obstrucción $y$ hernias). En Chile, se inicia su desarrollo a fines de los 90 y actualmente se encuentra disponible en la mayoría de los centros de salud públicos y privados $^{39}$.

Tomando una observación antigua, pero reemergente en oncología, como lo es, el rol de la inflamación en cáncer ${ }^{40,41}$, se estudió el valor pronóstico del índice sanguíneo de neutrófilo/ 
linfocito (NLR) como elemento predictivo en pacientes que van a cirugía curativa electiva en cáncer de colon en etapa II. De esta forma, un NLR $\geq 5$ resultó ser el único factor predictor de complicación, independiente de otros como el sexo, edad e invasión tumoral. Por otra parte, se estimó una mortalidad global de $26 \%$ en pacientes con NLR $<5$ y de $50 \%$ con NLR $\geq 5^{42}$.

Si bien es cierto que la metástasis hepática de origen colorrectal (MHCCR) es prácticamente otra enfermedad por sí misma, es importante considerar que al diagnóstico cerca de $20 \%$ de los CCR ya tiene MHCCR, siendo el único tratamiento efectivo la resección completa. Un estudio retrospectivo que incluyó a pacientes sometidos a una resección hepática por MHCCR entre 1990 y 2007, reveló que en un $86 \%$ se realizó 1 resección hepática, en tanto que en 8 (12\%) se debió efectuar una segunda hepatectomía debido a recurrencia. Se alcanzó una sobrevida global de 41 meses (38\% a 5 años), comparable con la literatura internacional, destacándose que esta conducta quirúrgica fue aumentando considerablemente en los últimos 9 años del estudio ${ }^{43}$.

En terapia sistémica, el 2015 se publicó una cohorte con 370 pacientes con cáncer de colon etapa II y III en un solo centro hospitalario (Hospital Clínico UC), demostrándose que el uso de quimioterapia adyuvante alcanza una sobrevida a 5 años cercana al $80 \%$ en pacientes etapa II y $74 \%$ para la etapa III, similar a estudios internacionales $^{44}$.

En 2018, el grupo del Dr. Nervi evaluó la respuesta de Regorafenib, un inhibidor multiquinasa oral, en una cohorte pequeña de 13 pacientes con CCR metastásico, encontrando una sobrevida global de 8,6 meses, sobrevida libre de enfermedad de 2,2 meses y efectos adversos como eritrodisistesia palmoplantar en el $23 \%$ y fatiga en $12 \%$ de los pacientes, resultados que son similares a los extranjeros y con tolerabilidad aceptable, que hacen de Regorafenib una alternativa plausible clínicamente, pero hasta la fecha inalcanzable en cuanto costos para nuestro sistema sanitario ${ }^{45}$.

Un área compleja en el tratamiento del CCR es la toma de decisiones de pacientes portadores de SL y PAF. Se debe definir entre una resección segmentaria o total de su colon y recto, considerando la calidad de vida del paciente y el mejor asesoramiento genético para sus familiares. En Chile se han constituido registros familiares y se han desarrollado equipos multidisciplinarios para el apoyo de estos pacientes en el sistema público y privado $^{46,47}$. En este sentido, en una alianza entre CLC, Clínica Alemana, la Pontificia Universidad Católica y la Universidad de Chile, se generó un Diplomado de Asesoramiento Genético en Síndromes Hereditarios que predisponen al cáncer en el año 2013. A la fecha se han realizado cuatro versiones en una plataforma e-learning destacando que un tercio de los graduados son de otros países de Latinoamérica.

\section{Conclusiones y Desafíos}

Las iniciativas en relación con el diagnóstico precoz del CCR en Chile gozan de una reciente historia. La capacitación entre 1992 y 1997 de los médicos del HSBA con apoyo de la Agencia de Cooperación Internacional del Japón (JICA), permitió mejorar sus técnicas endoscópicas y perfeccionarse en aspectos anatomopatológicos. En 2010, dichas colaboraciones chileno-niponas, se ampliaron en una alianza coordinada entre el MINSAL, CLC y UMDT, lo que dio origen al Programa de Prevención de Neoplasias Colorrectales (PRENEC), actualmente vigente, el que ha logrado perfeccionar capital humano avanzado (endoscopistas, patólogos digestivos, investigadores $\mathrm{MD} /$ $\mathrm{PhD}$ ), generar publicaciones, mantener 6 centros piloto a lo largo del país y comenzar a quebrar la curva de mortalidad por CCR en una de las zonas más afectadas por este tipo de cáncer, como lo es Magallanes (-20\%).

La detección precoz por TSDOi u otro método no invasivo, es una estrategia mundialmente exitosa para disminuir la mortalidad por CCR, pero la aplicación masiva en nuestro medio presenta aún barreras importantes, relacionadas con déficit de recurso humano, instrumentación e infraestructura. Sin embargo, el Plan Nacional de Cáncer, reconoce su efectividad y señala la necesidad de avanzar en el mediano plazo mediante "implementación progresiva" 48 .

Aun cuando se han desarrollado esfuerzos menos sistematizados respecto a la utilización de las nuevas clasificaciones moleculares y su relación con nuevas drogas, este conocimiento generado, nos debe llamar a buscar el porcentaje de KRAS mutado en nuestros pacientes con CCR. Esto, no solo permitiría optimizar clínicamente la indica- 
ción de las drogas biológicas, sino que además, este paradigma de la medicina de precisión, permitiría realizar planificación sanitaria moderna, a partir de nuestra propia epidemiología molecular, lo que podría "teóricamente" tener un efecto fármaco-económico positivo, sin embargo, hacen falta más estudios para dilucidar este intrincado punto $^{49,50}$.

En este artículo logramos recopilar múltiples experiencias chilenas en el ámbito de la investigación del CCR y en ellas pudimos visualizar que, (i) en las áreas de la historia natural de la enfermedad (CCR) existen esfuerzos que intentan comprender y optimizar el manejo del CCR en Chile; (ii) la generación de conocimiento se encuentra concentrada en universidades chilenas de la capital y centros públicos y privados asociados a estas y (iii) existe un vacío importante en relación con una organización ("task force") que aglutine todos los grupos de investigación en CCR y que pueda estar en sintonía con los cambios epidemiológicos y variaciones geográficas propias de la distribución del CCR en Chile.

Se reconoce que queda mucho por hacer y que los proyectos colaborativos nacionales-internacionales, las alianzas público-privadas, asistenciales-académicas, sectoriales-intersectoriales y la activa participación de las organizaciones de la sociedad civil, seguirán siendo necesarios.

Finalmente, no debemos olvidar que el CCR se asocia a factores de riesgo conocidos, entre ellos la dieta como el consumo de carnes rojas, carnes procesadas y alcohol y, por el contrario, siendo protector el adecuado consumo de frutas, verduras y leguminosas ${ }^{5}$. De este modo, es preciso avanzar significativamente en la promoción de estilos de vida saludables y en la implementación de acciones de prevención específica en los cánceres.

Agradecimientos: El Departamento de Manejo Integral de Cáncer del Ministerio de Salud agradece a la Embajada de Japón, a la Universidad Médica y Dental de Tokio y al Profesor Yoshinobu Eishi, $\mathrm{MD}, \mathrm{MedScD}, \mathrm{PhD}$, Chair del Departamento de Patología Humana de UDTM, quienes han ayudado de manera sistemática al Estado de Chile en el control de las Neoplasias Digestivas. Asimismo, los autores del trabajo agradecen especialmente a la Dra. Sylvia Santander, Jefa de la División de Prevención y Control de Enfermedades del MINSAL, por sus correcciones, comentarios y por alentarnos permanentemente a difundir los resultados sanitarios en el mundo académico.

\section{Referencias}

1. Aprueba Garantías Explícitas en Salud. Decreto número 4 [Internet]. 2013. Available from: http://www.supersalud.gob.cl/normativa/668/articles-8166_recurso_1.pdf

2. MINSAL. Guía de Práctica Clínica-Problema de Salud AUGE N ${ }^{\circ} 70$ Cáncer Colorrectal en personas de 15 años y más [Internet]. [citado el 18 de septiembre de 2019]. Available from: https://diprece.minsal.cl/garantias-explicitas-en-salud-auge-o-ges/guias-de-practica-clinica/ cancer-colorectal-en-personas-de-15-anos-y-mas/ recomendaciones/

3. Cruz Hernández JJ y colaboradores. Oncología Clínica. 6a Edición. 2017.

4. Brenner H, Kloor M, Pox CP. Colorectal cancer. Lancet (London, England) [Internet]. 2014; 383 (9927): 1490502. Available from: http://www.ncbi.nlm.nih.gov/ pubmed/24225001 [citado el 18 de septiembre de 2019].

5. Aicr, WCRF. Diet, Nutrition, Physical Activity and Cancer: a Global Perspective A summary of the Third Expert Report [Internet]. [citado el 23 de octubre de 2019]. Available from: http://gco.iarc.fr/today

6. Kuipers EJ, Grady WM, Lieberman D, Seufferlein T, Sung JJ, Boelens PG, et al. Colorectal cancer. Nat Rev Dis Prim [Internet]. 2015; 1 (1): 15065. Available from: http://www.nature.com/articles/nrdp201565 [citado el 18 de septiembre de 2019].

7. Global Cancer Observatory [Internet]. [citado el 11 de enero de 2019]. Available from: https://gco.iarc.fr/

8. Ministerio de Salud. Primer Informe de Registro Poblacionales de Cáncer de Chile del quinquenio 2007-2003 [Internet]. 2003 [citado el 11 de enero de 2019]. Available from: https://www.paho.org/chi/ index.php?option $=$ com_docman\&view=download\&alias=174-informe-rpc-chile-2003-2007\&category_slu$\mathrm{g}=$ cancer\&Itemid $=1145$

9. Series y Gráficos de Mortalidad-DEIS [Internet]. [citado el 11 de enero de 2019]. Available from: http://www. deis.cl/series-y-graficos-de-mortalidad/

10. Niechi I, Silva E, Cabello P, Huerta H, Carrasco V, Villar $\mathrm{P}$, et al. Colon cancer cell invasion is promoted by protein kinase CK2 through increase of endothelin-converting enzyme-1c protein stability. Oncotarget [Internet]. 2015; 6 (40): 42749-60. Available from: http://www. ncbi.nlm.nih.gov/pubmed/26543229 [citado el 18 de septiembre de 2019].

11. Silva-Pavez E, Villar P, Trigo C, Caamaño E, Niechi I, 
Pérez P, et al. CK2 inhibition with silmitasertib promotes methuosis-like cell death associated to catastrophic massive vacuolization of colorectal cancer cells. Cell Death Dis [Internet]. 2019; 10 (2): 73. Available from: http://www.nature.com/articles/s41419-019-1306-x [citado el 18 de septiembre de 2019].

12. De la Fuente López M, Landskron G, Parada D, Dubois-Camacho K, Simian D, Martínez M, et al. The relationship between chemokines CCL2, CCL3, and CCL4 with the tumor microenvironment and tumor-associated macrophage markers in colorectal cancer. Tumor Biol [Internet]. 2018; 40 (11): 101042831881005. Available from: http://www.ncbi.nlm.nih.gov/pubmed/30419802 [citado el 18 de septiembre de 2019].

13. Bellolio RF, Álvarez VK, Fuente L MD la, León GF, Fullerton MDA, Soto DG, et al. Cáncer colorrectal hereditario: análisis molecular de los genes APC y MLH1. Rev Med Chile [Internet]. 2006; 134 (7): 841-8. Available from: http://www.scielo.cl/scielo.php?script=sci_arttext\&pid=S0034-98872006000700006\&lng $=$ en\&nrm $=\mathrm{i}-$ so\&tlng=en [citado el 18 de septiembre de 2019].

14. Álvarez K, Hurtado C, Hevia MA, Wielandt AM, de la Fuente M, Church J, et al. Spectrum of MLH1 and MSH2 Mutations in Chilean Families With Suspected Lynch Syndrome. Dis Colon Rectum [Internet]. 2010; 53 (4): 450-9. Available from: http://www.ncbi.nlm.nih. gov/pubmed/20305446 [citado el 18 de septiembre de 2019].

15. Aravena T, Passalacqua C, Castillo Taucher S. Cáncer hereditario de colon: Aportes del diagnóstico genético molecular. Rev Med Chile [Internet]. 2010; 138 (12): 1530-4. Available from: http://www.scielo.cl/scielo.php?script=sci_arttext\&pi$\mathrm{d}=$ S0034-98872010001300009\&lng=en\&nrm=iso\&tln$\mathrm{g}=$ en [citado el 18 de septiembre de 2019].

16. Sveen A, Kopetz S, Lothe RA. Biomarker-guided therapy for colorectal cancer: strength in complexity. Nat Rev Clin Oncol [Internet]. 2019; 1-22. Available from: http://www.nature.com/articles/s41571-019-0241-1 [citado el 18 de septiembre de 2019].

17. Zhao B, Wang L, Qiu H, Zhang M, Sun L, Peng P, et al. Mechanisms of resistance to anti-EGFR therapy in colorectal cancer. Oncotarget [Internet]. 2017; 8 (3): 3980-4000. Available from: http://www.ncbi.nlm.nih.gov/ pubmed/28002810 [citado el 18 de septiembre de 2019].

18. Roa I, Sánchez T, Majlis A, Schalper K. Mutación del gen KRAS en el cáncer de colon y recto. Rev Med Chile [Internet]. 2013; 141 (9): 1166-72. Available from: http://www.scielo.cl/scielo.php?script=sci_arttext\&pi$\mathrm{d}=$ S0034-98872013000900009\&lng=en\&nrm=iso\&tln$\mathrm{g}=$ en [citado el 18 de septiembre de 2019].
19. Hurtado C, Wielandt AM, Zárate AJ, Kronberg U, Castro M, Yamagiwa K, et al. Análisis molecular del cáncer de colon esporádico. Rev Med Chile [Internet]. 2015; 143 (3): 310-9. Available from: http://www.scielo.cl/scielo.php?script=sci_arttext\&pi$\mathrm{d}=$ S0034-98872015000300005\&lng=en\&nrm=iso\&tln$\mathrm{g}=$ en [citado el 18 de septiembre de 2019].

20. Hurtado C, Encina G, Wielandt AM, Zárate AJ, Castro $\mathrm{M}$, Carrillo $\mathrm{K}$, et al. Prevalencia y características de mutaciones somáticas del gen KRAS en pacientes chilenos con cáncer colorrectal. Rev Med Chile [Internet]. 2014; 142 (11): 1407-14. Available from: http://www.scielo.cl/scielo.php?script=sci_arttext\&pi$\mathrm{d}=$ S0034-98872014001100007\&lng=en\&nrm=iso\&tln$\mathrm{g}=$ en [citado el 18 de septiembre de 2019].

21. Álvarez K, Orellana P, Villarroel C, Contreras L, Kawachi $\mathrm{H}$, Kobayashi $\mathrm{M}$, et al. EGFR pathway subgroups in Chilean colorectal cancer patients, detected by mutational and expression profiles, associated to different clinicopathological features. Tumor Biol [Internet]. 2017; 39 (9): 101042831772451. Available from: http:// www.ncbi.nlm.nih.gov/pubmed/28936923 [citado el 18 de septiembre de 2019].

22. Sole CV, Calvo FA, Ferrer C, Álvarez E, Carreras JL, Ochoa E. Human cytomegalovirus and Epstein-Barr virus infection impact on 18F-FDG PET/CT SUVmax, CT volumetric and KRAS-based parameters of patients with locally advanced rectal cancer treated with neoadjuvant therapy. Eur J Nucl Med Mol Imaging [Internet]. 2015; 42 (2): 186-96. Available from: http://www.ncbi.nlm. nih.gov/pubmed/25269837 [citado el 18 de septiembre de 2019].

23. Wielandt AM, Villarroel C, Hurtado C, Simian D, Zamorano D, Martínez M, et al. Caracterización de pacientes con cáncer colorrectal esporádico basado en la nueva subclasificación molecular de consenso. Rev Med Chile [Internet]. 2017; 145 (4): 419-30. Available from: http://www.scielo.cl/scielo.php?script=sci_arttext\&pi$\mathrm{d}=$ S0034-98872017000400001\&lng=en\&nrm=iso\&tln$\mathrm{g}=$ en [citado el 18 de septiembre de 2019].

24. Kronberg U. Estrategias de prevención y detección precoz del cáncer de colon y recto en individuos de riesgo promedio y alto riesgo. Rev Médica Clínica Las Condes [Internet]. 2011; 22 (4): 493-8. Available from: https://www.sciencedirect.com/science/article/ pii/S0716864011704552 [citado el 18 de septiembre de 2019].

25. Francisco LK. Actualizaciones en el diagnóstico y tratamiento quirúrgico de los pacientes con cáncer de colon. Rev Médica Clínica Las Condes [Internet]. 2013; 24 (4): 645-53. Available from: https://linkinghub.elsevier. 
com/retrieve/pii/S0716864013702037 [citado el 18 de septiembre de 2019].

26. Silva-Illanes N, Espinoza M. Critical Analysis of Markov Models Used for the Economic Evaluation of Colorectal Cancer Screening: A Systematic Review. Value Heal [Internet]. 2018; 21 (7): 858-73. Available from: https://www.valueinhealthjournal.com/article/S10983015(18)30182-7/fulltext?_returnURL=https\%3A\%2F\%2Flinkinghub.elsevier.com $\% 2$ Fretrieve $\% 2$ Fpii $\%$ 2FS1098301518301827\%3Fshowall\%3Dtrue [citado el 18 de septiembre de 2019].

27. López-Köstner F, Kronber U, Zárate AJ, Wielandt AM, Pinto E, Suazo C, et al. Programa de detección de neoplasias colorrectales en población mayor de 50 años. Rev Med Chile [Internet]. 2012; 140 (3): 281-6. Available from: http://www.scielo.cl/scielo.php?script=sci_arttext\&pid=S0034-98872012000300001\&lng=en\&nrm=iso\&tlng=en [citado el 18 de septiembre de 2019].

28. Okada T, Tanaka K, Kawachi H, Ito T, Nishikage T, Odagaki $\mathrm{T}$, et al. International collaboration between Japan and Chile to improve detection rates in colorectal cancer screening. Cancer [Internet]. 2016; 122 (1): 71-7. Available from: http://www.ncbi.nlm.nih.gov/ pubmed/26445309 [citado el 18 de septiembre de 2019].

29. Ito T, Kawachi H, Peñaloza P, Zárate AJ, Ponce A, Kobayashi M, et al. Protocolo Estandarizado de Anatomía Patológicade lesiones polipoideas en Proyecto de Prevención de Neoplasias Colorrectales (PRENEC) en Chile. Gastroenterol latinoam 2016; 27 (1): 37-46 [Internet]. Available from: http://webcache.googleusercontent.com/search?q=cache:CtjPdiF4GlAJ:gastrolat. org/DOI/PDF/10.0716/gastrolat2016n100005.pdf+\&c$\mathrm{d}=1 \& \mathrm{hl}=\mathrm{es} \& \mathrm{ct}=\mathrm{clnk} \& \mathrm{gl}=\mathrm{cl}$ [citado el 18 de septiembre de 2019].

30. Kobayashi M, Kawachi H, Pasternak S, Delgado C, Pinto $\mathrm{P}$, Ito $\mathrm{T}$, et al. Histopathologic study from a colorectal cancer screening in Chile. Eur J Cancer Prev [Internet]. 2019; 28 (4): 245-53. Available from: http://www.ncbi. nlm.nih.gov/pubmed/29958195 [citado el 18 de septiembre de 2019].

31. López-Kostner F, Zárate AJ, Ponce A, Kronberg U, Kawachi H, Okada T, et al. Programa multicéntrico de cribado de cáncer colorrectal en Chile. Rev Med Chile [Internet]. 2018; 146 (6): 685-92. Available from: http://www.scielo.cl/scielo.php?script=sci_arttext\&pi$\mathrm{d}=$ S0034-98872018000600685\&lng=en\&nrm=iso\&tln$\mathrm{g}=$ en [citado el 18 de septiembre de 2019].

32. Wielandt AM, Zárate AJ, Hurtado C, Orellana P, Álvarez K, Pinto E, et al. Síndrome de Lynch: selección de pacientes para el estudio genético mediante análisis de inestabilidad microsatelital e inmunohistoquímica. Rev
Med Chile [Internet]. 2012; 140 (9): 1132-9. Available from: http://www.scielo.cl/scielo.php?script=sci_arttext\&pid=S0034-98872012000900005\&lng=en\&nrm=iso\&tlng=en [citado el 18 de septiembre de 2019].

33. Bannura CG, Cumsille G Ma, Barrera EA, Contreras PJ, Melo LC, Soto CD, et al. Reoperaciones precoces en cirugía colorrectal: Análisis uni y multivariado de factores de riesgo. Rev Chil Cir 2007; 59 (4): 281-6.

34. Bannura CG, Gallardo VC, Vargas RC, Barrera EA, Melo LC, Illanes FF. Tasa de reingreso no programado en cirugía colorrectal electiva. Rev Chil Cir 2015; 67 (4): 393-8.

35. Bannura CG, ACGM, Barrera EA, Contreras PJ, Melo LC, Soto CD. Resultados del tratamiento quirúrgico del cáncer de colon. Análisis de 439 pacientes. Rev Chil Cir 2010; 62 (5): 491-6.

36. Bannura CG, Barrera EA, Melo LC, Contreras PJ, Soto CD. Resultados del tratamiento con neoadyuvancia del cáncer de recto localmente avanzado. Rev Chil Cir 2010; 62 (6): 607-13.

37. Bannura CG, Barrera EA, Melo LC, Illanes FF, Gallardo VC, Suazo LC. Cecostomía amplia: Una alternativa de protección de una anastomosis colorrectal baja en cáncer de recto. Rev Chil Cir 2014; 66 (4): 345-50.

38. Abedrapo MM, López SN, Azolas RM, Díaz MB, Sanguineti AM, Llanos JLB, et al. Resección total del mesorrecto por vía transanal en cáncer de recto. Análisis de resultados de una serie preliminar en un hospital universitario. Rev Chil Cir 2017; 69 (1): 53-9.

39. López Köstner F, Zárate A, León F, García-Huidobro MA, Bellolio F, Pinedo G, et al. Resultados preliminares de la cirugía laparoscópica del cáncer colorrectal. Rev Chil Cir. [Internet]. 2006 [citado el 23 de septiembre de 2019]; 58 (2): 106-13. Available from: http://www.scielo.cl/scielo.php?script=sci_arttext\&pi$\mathrm{d}=$ S0718-40262006000200006\&lng=en\&nrm=iso\&tln$\mathrm{g}=\mathrm{en}$.

40. Balkwill F, Mantovani A. Inflammation and cancer: Back to Virchow? Vol. 357, Lancet. Elsevier Limited; 2001. p. 539-45.

41. Greten FR, Grivennikov SI. Inflammation and Cancer: Triggers, Mechanisms, and Consequences. Immunity 2019; 51 (1): 27-41.

42. Urrejola GI, Bambs CE, Espinoza MA, Gellona J, Zúñiga ÁM, Molina ME, et al. Un índice neutrófilo/linfocito elevado se asocia a peor pronóstico en cancer de colon etapa II resecado. Rev Med Chile [Internet]. 2013; 141 (5): 602-8. Available from: http://www.scielo.cl/scielo.php?script=sci_arttext\&pi$\mathrm{d}=$ S0034-98872013000500008\&lng=en\&nrm=iso\&tln$\mathrm{g}=$ en [citado el 18 de septiembre de 2019]. 
43. Norero E, Jarufe N, Butte JM, Norero B, Duarte I, Torres J, et al. Resultados del tratamiento quirúrgico de las metástasis hepáticas por cáncer colorrectal. Rev Med Chile [Internet]. 2009; 137 (4): 487-96. Available from: http://www.scielo.cl/scielo.php?script=sci_arttext\&pi$\mathrm{d}=$ S0034-98872009000400006\&lng=en\&nrm=iso\&tln$\mathrm{g}=$ en [citado el 18 de septiembre de 2019].

44. Mondaca S, Villalón C, Leal JL, Zúñiga Á, Bellolio F, Padilla $\mathrm{O}$, et al. Beneficio de la quimioterapia adyuvante en pacientes con cáncer de colon: cohorte retrospectiva de un hospital clínico universitario. Rev Med Chile [Internet]. 2016; 144 (2): 145-51. Available from: http://www.scielo.cl/scielo.php?script=sci_arttext\&pi$\mathrm{d}=$ S0034-98872016000200001\&lng=en\&nrm=iso\&tln$\mathrm{g}=$ en [citado el 18 de septiembre de 2019].

45. Leal JL, Briones J, Herrera ME, Müller B, Nervi B, Mondaca S. Regorafenib adjusted dose for Chilean patients with chemoresistant metastatic colorectal cancer: a case series. Ecancermedicalscience [Internet]. 2018; 12. Available from: https://ecancer.org/journal/12/full/875-regorafenib-adjusted-dose-for-chilean-patients-with-chemoresistant-metastatic-colorectal-cancer-a-case-series.php [citado el 18 de septiembre de 2019].

46. Vaccaro CA, Sarroca C, Rossi B, López-Kostner F, Domínguez M, Calo NC, et al. Lynch syndrome in South
America: past, present and future. Fam Cancer [Internet]. 2016 Jul 23 [citado el 23 de septiembre de 2019]; 15 (3): 437-45. Available from: http://link.springer. com/10.1007/s10689-016-9903-7.

47. Vaccaro CA, López-Kostner F, Adriana DV, Palmero EI, Rossi BM, Antelo M, et al. From colorectal cancer pattern to the characterization of individuals at risk: Picture for genetic research in Latin America. Int J Cancer [Internet]. 2019; 145 (2): 318-26. Available from: http://doi.wiley.com/10.1002/ijc.31920 [citado el 23 de septiembre de 2019].

48. Ministerio de Salud G de C. Plan Nacional de Cáncer 2018-2028 [Internet]. [citado el 20 de julio de 2019]. Available from: https://www.minsal.cl/wp-content/uploads/2019/01/2019.01.23_PLAN-NACIONAL-DE-CANCER_web.pdf.

49. Wright SJ, Newman WG, Payne K. Accounting for Capacity Constraints in Economic Evaluations of Precision Medicine: A Systematic Review. Vol. 37, PharmacoEconomics. Springer International Publishing; 2019. p. 1011-27.

50. Love-Koh J, Peel A, Rejon-Parrilla JC, Ennis K, Lovett R, Manca A, et al. The Future of Precision Medicine: Potential Impacts for Health Technology Assessment. Pharmacoeconomics 2018; 36 (12): 1439-51. 